\title{
Relationship between cooperation in an iterated prisoner's dilemma game and the discounting of hypothetical outcomes
}

\author{
RICHARD YI, MATTHEW W. JOHNSON, and WARREN K. BICKEL \\ University of Vermont, Burlington, Vermont
}

\begin{abstract}
A number of authors have proposed that preference for a larger, delayed reward in delay discounting is similar to cooperation in a repeated prisoner's dilemma game versus tit-for-tat. This proposal was examined by correlating delay-discounting (Experiment 1) and probability-discounting (Experiment 2) rates for hypothetical monetary gains and losses with performance in a repeated prisoner's dilemma game. Correlations between rate of delay discounting (discounting parameters and area under the curve) and proportion of cooperation in the repeated prisoner's dilemma game versus tit-for-tat were significant across three magnitudes, and correlations were generally higher with discounting for losses than with that for gains. As was expected, correlations between rate of delay discounting and performance versus a random strategy in the prisoner's dilemma game were not significant. Correlations between rate of probability-discounting and cooperation rate in a repeated prisoner's dilemma game versus neither a tit-for-tat nor a random strategy were significant.
\end{abstract}

Delay-discounting procedures model impulsiveness as a preference for small, immediate rewards over larger, delayed rewards. Self-control, on the other hand, is indicated by a preference for larger, delayed rewards over small, immediate rewards. Although most real-life choice situations do not involve such distinct alternatives, the evidence that delay-discounting procedures are a good analogue to impulsivity/self-control situations is compelling. Populations generally thought to differ in impulsiveness are differentiated with delay-discounting procedures: Individuals at high risk for impulsive behavior (on the basis of DSM-IV diagnoses) exhibit more delay discounting than do those at low risk (Crean, de Wit, \& Richards, 2000), and younger individuals exhibit more delay discounting than do older individuals (Green, Fry, \& Myerson, 1994). Delay-discounting rate has been significantly correlated with manifestations of impulsivity, including the age at which alcohol, cigarettes, and marijuana are first used, as well as the total number of illicit drugs used (Kollins, 2003). Furthermore, pathological gamblers (Petry, 2001b), cocaine dependents (Coffey, Gudleski, Saladin, \& Brady, 2003), opioid dependents (Kirby, Petry, \& Bickel, 1999; Madden, Petry, Badger, \& Bickel, 1997), problem drink-

This research was conducted at the University of Vermont and was funded by National Institute on Drug Abuse Grants R01 DA11692 and T32 DA07242. The first and third authors are now located at the University of Arkansas for Medical Sciences in Little Rock. The second author is located at Johns Hopkins University in Baltimore. The authors thank Amy Prue and Kirstin Gatchalian for their assistance with data collection and manuscript preparation. Correspondence concerning this article should be addressed to R. Yi, Department of Psychiatry, College of Medicine, University of Arkansas for Medical Sciences, 4301 West Markham Street, \#843, Little Rock, AR 72205 (e-mail: ryi@uams.edu). ers (Petry, 2001a; Vuchinich \& Simpson, 1998), and cigarette smokers (Baker, Johnson, \& Bickel, 2003; Bickel, Odum, \& Madden, 1999; Mitchell, 1999) all exhibit more delay discounting than do controls.

A typical delay-discounting procedure determines an indifference point, the present value of an outcome that an individual judges to be subjectively equivalent to a specific amount that will be delayed by a specific time period. An indifference point is determined for each of numerous delays for that amount, and an indifference curve is determined. Mazur's (1987) hyperbolic discounting equation (Equation 1) works well when fit to these data, accounting for a high percentage of the variance:

$$
v_{\mathrm{d}}=\frac{V}{1+k d} \text {. }
$$

In this equation, the discounted value of an outcome $\left(v_{\mathrm{d}}\right)$ is equal to the ratio of the undiscounted value $(V)$ to the quantity of one plus delay $(d)$ multiplied by a discounting parameter $(k)$. This free parameter $(k)$ provides a measure of the tendency to prefer the smaller, immediate reward to the larger, delayed reward.

Ainslie (1992) and Rachlin (2000) have proposed that self-control in delay discounting is similar to cooperation in a repeated prisoner's dilemma game (PDG), versus a titfor-tat (TFT) strategy; both require an individual to forgo the best immediate outcome for an even better, delayed outcome. A PDG is a mixed-motive strategy game played with two participants. Figure 1 is a diagram of the choice alternatives (axes) and outcomes (cells) of a typical PDG. Each player has two choice alternatives (cooperation and defection), and the outcome of each player's choice depends on both his or her own choice and the other player's choice. Defection results in the best outcome for each 


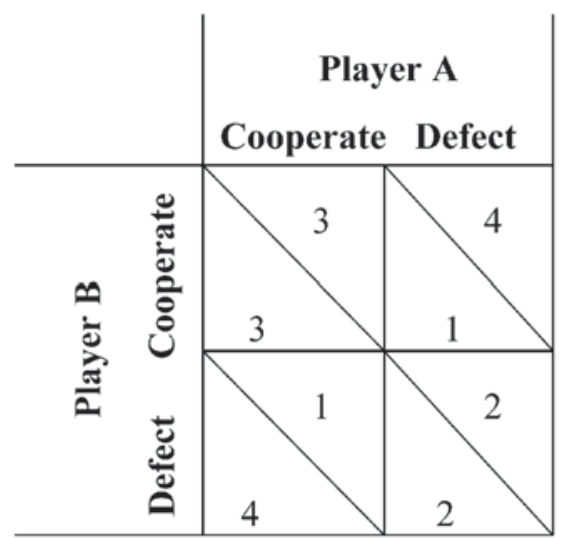

Figure 1. Choice alternatives (axes) and outcomes (cells) for a prisoner's dilemma game.

player (4 units rather than $3 ; 2$ units rather than 1), regardless of the other player's choice. However, when both players use this reasoning and defect, both of them receive a lower value outcome ( 2 units each) than they would have had they cooperated (3 units each).

In an iterated PDG (IPG), the PDG is repeated over numerous trials with the same two participants. In such a situation, the optimal strategy (to earn the highest cumulative result) depends on the strategy applied by the other player. TFT is a reciprocal strategy in which the player using the strategy cooperates on the first trial and then reciprocates the other player's previous choice on successive trials. For instance, cooperation by Player A in Trial 1 is reciprocated by a cooperation by Player $\mathrm{B}$ (applying the TFT strategy) in Trial 2, and so on. A string of choices by Player A (CDDDCCDC) in IPG would be reciprocated by Player $\mathrm{B}$ with $\mathrm{CCDDDCCD}$, where $\mathrm{C}=$ cooperation and $\mathrm{D}=$ defection. The optimal strategy when opposing a player applying a TFT strategy is to cooperate on all the trials. Exclusive cooperation is optimal because cooperating on trial $n$ leaves the player with the best alternatives ( 4 or 3 units in Figure 1) in trial $n+1$ (resulting from the reciprocal cooperation by the TFT player on the next trial). A defection on trial $n$ results in a higher outcome for that trial (4 units instead of 3 units, 2 units instead of 1 unit) but leaves the player with the worst alternatives ( 2 or 1 units) in trial $n+1$. Exclusive cooperation results in a mean outcome per trial of 3 units, exclusive defection results in a mean of 2 units, and alternation of cooperation and defection results in a mean of 2.5 units [ 4 units + 1 unit) / 2 trials]. To maximize overall outcome with a TFT opponent, the player must consistently cooperate, choosing the smaller of the two available outcomes in the current trial.

Harris and Madden (2002) examined Ainslie (1992) and Rachlin's (2000) hypothesis directly by correlating the delay-discounting rate of college students and their performance in an IPG. Following a delay-discounting procedure for a real $\$ 10$ delayed amount, the participants played a 40-trial IPG against a computer applying a TFT strategy. Delay-discounting parameters were obtained using Equation 1, and as was predicted, discounting parameters and percentages of defection in the IPG were significantly correlated, with high discounters tending to defect more.

\section{EXPERIMENT 1}

In the present study, we attempted to expand on the findings of Harris and Madden (2002), with a number of important differences. Foremost was the inclusion of a control condition. In Harris and Madden, the participants played the IPG only against a TFT player. Because no other IPG strategy was examined, it is unclear whether the participants' cooperation rates were sensitive to the IPG strategy used by the computer player. Without a control condition, an alternative explanation is that rate of delay discounting is related to a generalized tendency to cooperate in an IPG regardless of the other player's strategy; those who delay discount at high rates may be more likely to defect in an IPG against any strategy. A nonsignificant correlation between delay-discounting rate and cooperation when the opponent uses a non-TFT IPG strategy is necessary to conclude that self-control (in delay discounting) is similar to cooperation (vs. TFT in an IPG). A random strategy (RAN), where cooperation/defection was selected randomly from trial to trial, was included as a second IPG strategy. In addition, the present study included dependent measures not collected or reported in Harris and Madden: Discounting parameters were obtained for three magnitudes of gains and losses, as were demographic variables that may affect performance in an IPG (e.g., IQ). The present procedure followed an order different from that in Harris and Madden, with the IPG preceding the delay-discounting procedure. Finally, the participants in the present study were not informed about the computer strategy in the IPG before the experimental session, as they were in Harris and Madden.

In accord with the results in the previous literature (see Green \& Myerson, 2004, for a review), magnitude and sign effects were expected; large-magnitude outcomes and gains should be discounted less than small-magnitude outcomes and losses, respectively. A significant negative correlation was expected between rate of delay discounting and cooperation rate in the IPG in the TFT condition; no correlation was expected with cooperation rate in the RAN condition.

\section{Method}

Participants. Thirty-one college students, between 18 and 23 years of age, were initially recruited. One participant (18-year-old male) failed to complete both sessions and was excluded from all analyses. The mean age of the remaining 30 participants ( 12 of them male) was 19 years. All the participants were recruited from the psychology department participant pool and received credit in an introductory-level class for participation.

Apparatus and Instruments. The Quick Test (QT; Ammons \& Ammons, 1962) has been found to be a good instrument for measuring general intelligence in a short period of time (Joesting \& Joesting, 1971). The QT requires the participant to identify one picture (out of four) that best matches the meaning of each of 50 words. 
Both the IPG and the delay-discounting measures were obtained using a laptop computer running Visual Basic 6.0.

Procedure. Each participant attended two experimental sessions, with the only requirement being that they should occur on different days. The QT was administered before any other procedures in the first session. Immediately after administration of the QT, the participants began the IPG.

The directions page of the IPG was the following:

You will be playing a game for hypothetical money where you and the computer will make simultaneous choices. On each trial, you will be asked to choose either "cooperation" or "defection." At the same time that you make your choice, the computer will also choose "cooperation" or "defection." The amount of money you earn on each trial will depend on the combination of your choice and the computer's choice.

\begin{tabular}{lll} 
Computer's Choice & Your Choice & Your Earnings \\
\hline Cooperation & Defection & $\$ 25$ \\
Cooperation & Cooperation & $\$ 20$ \\
Defection & Defection & $\$ 10$ \\
Defection & Cooperation & $\$ 5$
\end{tabular}

You will not know the choice of the computer before you make your choice. Following each trial, you will be informed of the computer's choice, the amount that you earned on that trial, and the total earnings you have accumulated to that point.

The participants started the IPG after they had had an opportunity to ask questions. The choice screen appeared after the participants clicked on the "Begin" button. On the choice screen in each trial, the "Cooperate" and "Defect" alternatives were presented in rectangular command boxes to the left and right of center, respectively. Below these was the phrase "Please make your choice now." Once the participant had clicked on one of the alternatives, the feedback page appeared on the screen and said "You chose cooperate (or defect) on this trial. The computer chose cooperate (or defect) on this trial. You earn $\$$ on this trial, and $\$$ in this game so far." After

$10 \mathrm{sec}$, the next trial began with the choice page.

The computer could apply one of two strategies in the IPG: TFT or RAN. In the TFT condition, the computer cooperated on the first trial and mimicked the player's choice on the previous trial thereafter. A player's cooperation was reciprocated with a cooperation by the computer on the following trial; a defection by the player was reciprocated by the computer with a defection on the following trial. In the RAN condition, the computer chose cooperation or defection with a probability of .5 on each trial. Unlike play against the TFT strategy, defection is the optimal strategy against RAN, since the player cannot affect the computer's future choices.

The IPG in the two sessions consisted of 60 trials each and differed in the strategy applied by the computer: The computer applied the TFT strategy in one session and the RAN strategy in the other. Their order was counterbalanced, with 15 participants each playing against TFT or RAN in the first session. The delay-discounting procedure followed the IPG in all sessions. The outcomes from choices in the IPG were hypothetical.

To obtain measures of delay discounting, a computerized discounting procedure was employed (see Johnson \& Bickel, 2002, for a thorough review). For each of three magnitudes (of the larger, delayed outcome: $\$ 10, \$ 100, \$ 1,000)$, the participants chose between two hypothetical outcomes in each trial: a smaller, immediate outcome that adjusted from trial to trial (on the basis of the programmed algorithm) and a larger, delayed outcome that remained constant. Indifference points were obtained for gains at each of the following delays: 1 day, 1 week, 1 month, 6 months, 1 year, 5 years, and 25 years, with delays always presented in increasing order. The same order and procedure were then applied with the hypothetical outcomes for losses instead of gains: $\$ 10, \$ 100$, and $\$ 1,000$. An attempt was made to counterbalance the order in which the three magnitudes were presented (ascending and descending) with the order of IPG strategy (TFT or RAN first), although perfect counter- balancing was not accomplished because the number of participants was not divisible by the number of possible orderings (four). The delay-discounting procedures in the two experimental sessions were identical in all respects.

\section{Results}

Mean proportion of cooperation was higher against TFT (.51) than against RAN $[.34 ; F(1,29)=16.64, p<.05]$. These proportions were comparable within the first 30 trials $[M=.44$ and .43 for TFT and RAN, respectively; $F(1,29)=0.12, p>.05]$ but diverged in the last 30 trials $[M=.58$ and .25 for TFT and RAN, respectively; $F(1,29)=$ $38.28, p<.05]$. The hypothetical earnings against TFT $(M=\$ 933.11)$ and RAN $(M=\$ 962.67)$ were not significantly different $[F(1,29)=2.06, p>.05]$. Table 1 shows Pearson correlations between age, QT score, proportion of cooperation, and money earned against in each IPG condition. Gender was not significantly correlated with any dependent variables. Measures obtained from the IPG were significantly correlated with neither age nor QT. They were, however, significantly correlated with each other. Significantly positive correlations in cooperation rates between TFT and RAN indicate some tendency to cooperate regardless of the IPG strategy.

Nonlinear estimation with Statistica 5.0 was used to determine delay-discounting parameters $(k)$ with median indifference points in the two IPG conditions for the three magnitudes and two signs according to Equation 1 (shown with goodness of fit in Figure 2). Because fits to individuals' data were generally poor, area-under-thecurve (AUC; Myerson, Green, \& Warusawitharana, 2001) measures were also calculated to confirm/disconfirm the findings with discounting parameters. The AUC measure does not require any assumptions regarding the shape of the discounting function and can address reservations regarding the validity of the obtained parameters. Spearman correlation analyses were conducted between discounting parameters and AUC measures. All the correlations were negative (as would be predicted) and were significant at $p=.000001$. In fact, the lowest correlation was -.93 , with most correlations being greater than -.95 . The results of these correlations indicate that the obtained dis-

TABLE 1

Pearson Correlations Between Demographic Variables and Iterated Prisoner's Dilemma Game Data (Experiment 1)

\begin{tabular}{|c|c|c|c|c|c|c|}
\hline & \multirow[b]{2}{*}{ Age } & \multirow{2}{*}{$\begin{array}{c}\text { Quick } \\
\text { Test }\end{array}$} & \multicolumn{2}{|c|}{ TFT } & \multicolumn{2}{|c|}{ RAN } \\
\hline & & & Earn & Coop & Earn & Coop \\
\hline Age & - & .34 & .23 & .23 & -.01 & -.01 \\
\hline Quick test & - & - & .11 & .10 & .27 & -.20 \\
\hline \multicolumn{7}{|l|}{ TFT } \\
\hline Earn & - & - & - & $.99^{*}$ & $-.40^{*}$ & $.42^{*}$ \\
\hline Coop & - & - & - & - & $-.39^{*}$ & $.42^{*}$ \\
\hline \multicolumn{7}{|l|}{ RAN } \\
\hline Earn & - & - & - & - & - & $-.75^{*}$ \\
\hline Coop & - & - & - & - & - & - \\
\hline
\end{tabular}

Note-TFT, tit-for-tat strategy; RAN, random strategy; Earn, money earned; Coop, proportion of cooperation. ${ }^{*} p<.05$. 

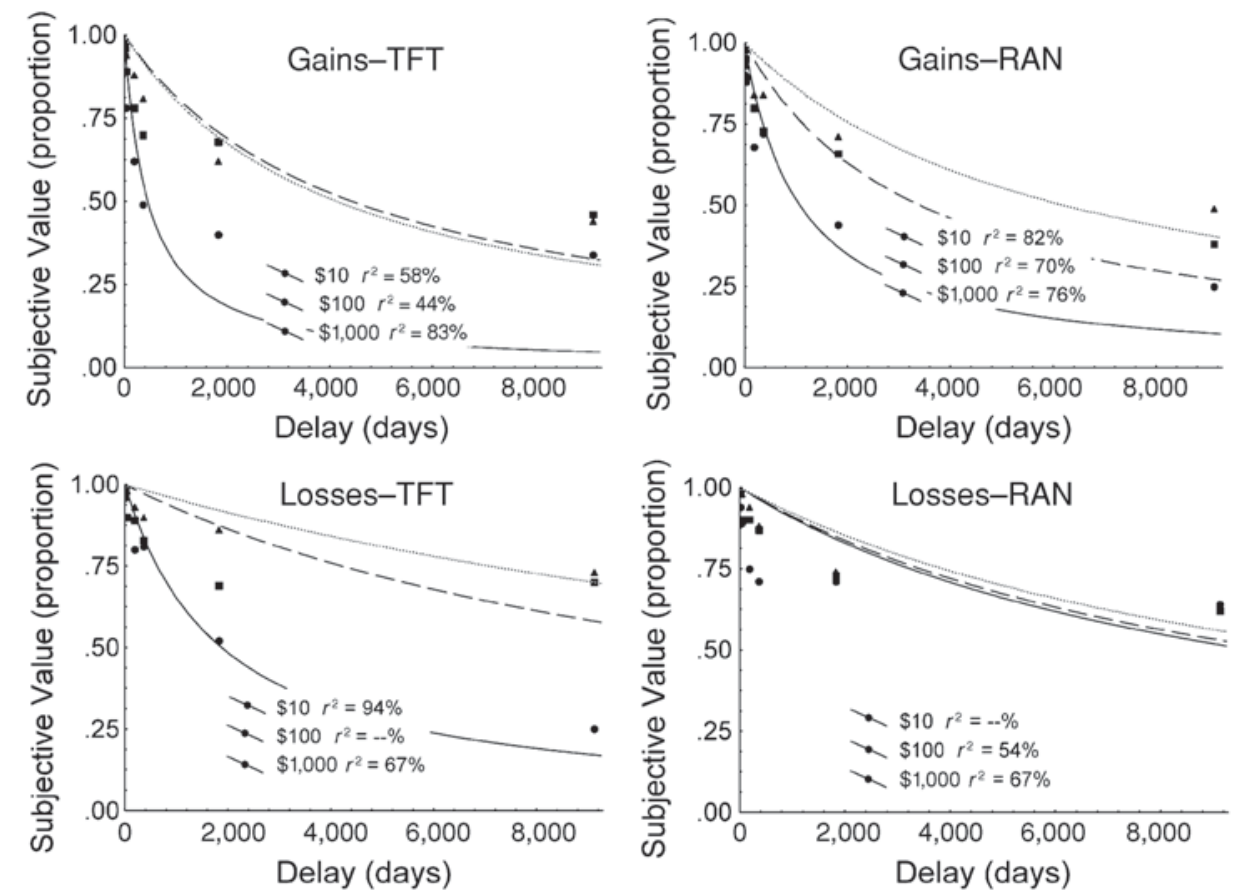

Figure 2. Median indifference points fitted to Equation 1. The graphs show (clockwise from top left) gains in the tit-for-tat (TFT) strategy, gains in the random (RAN) strategy, losses in the RAN strategy, and losses in the TFT strategy conditions. Different magnitudes are represented in each graph.

counting parameters are as indicative of rate of discounting as is AUC.

Twelve discounting parameters were calculated for each participant ( 2 IPG conditions $\times 2$ signs $\times 3$ magnitudes), and natural logarithm transformations were conducted to normalize the distributions of parameters for the purpose of parametric analyses. Order effects of IPG condition (i.e., TFT in first or second session) were ruled out with an analysis of variance (ANOVA), and the remaining analyses pooled data across order of IPG. A $2 \times 2 \times 3$ repeated measures ANOVA was conducted on discounting parameters. No difference was found in the delay-discounting rate as a result of IPG condition $[F(1,29)=0.01, p>.05]$ or sign $[F(1,29)=0.46, p>.05]$. A significant difference was observed in the magnitude condition $[F(2,58)=12.92$, $p<.05]$, and post hoc analyses revealed significant differences between the $\$ 10(M=-7.02)$ and the remaining magnitudes ( $\$ 100 M=-8.02, \$ 1,000 M=-8.59)$. The results of an ANOVA with AUC measures confirmed the analysis with discounting parameters. There was a similar effect of magnitude, but no effects of condition or sign were observed. The nonsignificant effect of sign was in the predicted direction at $p=.06$. Planned comparisons of the sign condition at each magnitude indicated a significant effect at the $\$ 10$ magnitude, but not at the others.

Spearman correlation analyses were conducted between discounting parameters in the TFT and RAN conditions, to determine the consistency of the discounting parameter across sessions. All the relevant correlations (e.g., \$10 gains in TFT and $\$ 10$ gains in RAN) were moderate to high (between .58 and .82 ) and were statistically significant at $p=.001$. Spearman correlations were calculated between performance measures for the IPG (total proportion of cooperation, proportion of cooperation in the first half of the session, and proportion of cooperation in the last half of the session) and delay-discounting parameters. Table 2 displays the correlation matrix between these measures obtained in the TFT and RAN conditions. One outlier who defected on all IPG trials was excluded from

Table 2

Spearman Correlations Between Iterated Prisoner's Dilemma Game Performance and Delay-Discounting Parameters

\begin{tabular}{cllcc}
\hline Strategy & \multicolumn{1}{c}{ Outcome } & $\begin{array}{c}\text { Total } \\
\text { Coop }\end{array}$ & $\begin{array}{c}\text { Trials 1-30 } \\
\text { Coop }\end{array}$ & $\begin{array}{c}\text { Trials 31-60 } \\
\text { Coop }\end{array}$ \\
\hline TFT & \$10 gains & -.27 & -.17 & -.31 \\
& \$100 gains & $-.37^{*}$ & -.31 & $-.40^{*}$ \\
& \$1,000 gains & $-.39^{*}$ & -.32 & $-.44^{*}$ \\
& \$10 losses & $-.57^{* *}$ & $-.50^{* *}$ & $-.50^{* *}$ \\
& \$100 losses & $-.65^{* *}$ & $-.66^{* *}$ & $-.55^{* *}$ \\
RAN & \$1,000 losses & $-.54^{* *}$ & $-.59^{* *}$ & $-.45^{*}$ \\
& \$10 gains & -.19 & $-.44^{*}$ & -.03 \\
& \$100 gains & -.01 & -.27 & .12 \\
& \$1,000 gains & -.01 & -.30 & .14 \\
& \$10 losses & -.14 & -.23 & -.08 \\
& \$100 losses & -.25 & -.33 & -.15 \\
& \$1,000 losses & -.05 & -.06 & -.16 \\
\hline
\end{tabular}

Note-TFT, tit-for-tat; RAN, random; Coop, proportion of cooperation. ${ }^{*} p<.05 .{ }^{* *} p<.01$. 

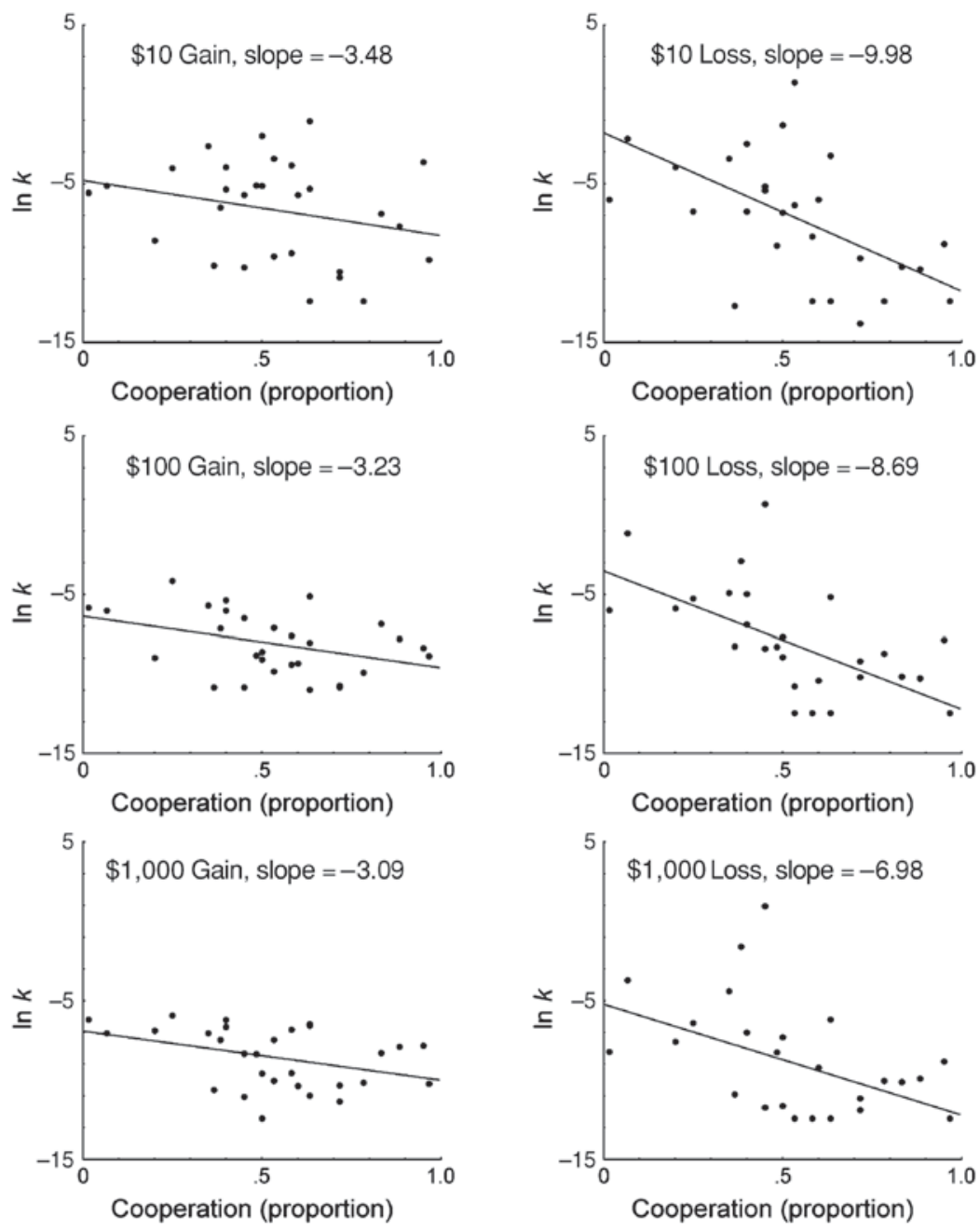

Figure 3. Scatterplots of natural logarithm transformed $\boldsymbol{k}$ as a function of proportion of cooperation against the tit-for-tat strategy, with linear regression lines.

these correlations. This participant was insensitive to both delay and probability in the discounting procedures, resulting in indifference points that were always equal to the delayed and probabilistic amounts. This pattern suggests that the participant did not attend to the available alternatives. Figure 3 displays scatterplots of the proportion of cooperation and the natural logarithm of the discounting parameter $(\ln k)$ for gains and losses in the TFT condition. Linear regression lines were fit to the data, and slopes are reported. Delay-discounting parameters and performance in the IPG were all negatively correlated, with the highest correlations obtained with discounting for losses. With the exception of the $\$ 10$ gains condition, all the correlations involving total proportion of cooperation were statistically significant at $p=.05$. Figure 4 displays scatterplots of the proportion of cooperation and $\ln k$ for gains and losses in the RAN condition. Most of the correlations between discounting parameters and performance in the IPG were negative, although none was significant. Spearman correlations between AUC measures and proportion of cooperation against TFT and RAN (like the analysis with $k$ in Table 2) were very similar to the results obtained with discounting parameters. AUC measures obtained in discounting for losses were significantly correlated with performance against TFT, with no significant correlations with performance against RAN. Unlike discounting parameters from the $\$ 100$ and $\$ 1,000$ gains conditions (which were marginally significant), AUC measures from those conditions and performance in the IPG were not statistically significant.

\section{Discussion}

The relationship proposed by Ainslie (1992) and Rachlin (2000) and supported by Harris and Madden (2002) was the similarity of cooperative behavior versus a TFT strategy in the IPG to choice of the larger, delayed reward in delay discounting (self-control). Table 2 and Figures 3 and 4 combine to form a strong argument for this relationship. The present results verify Harris and Madden's findings and extend them in a number of ways. First and foremost is the nonsignificant relationship between performance in the IPG against a non-TFT strategy and 

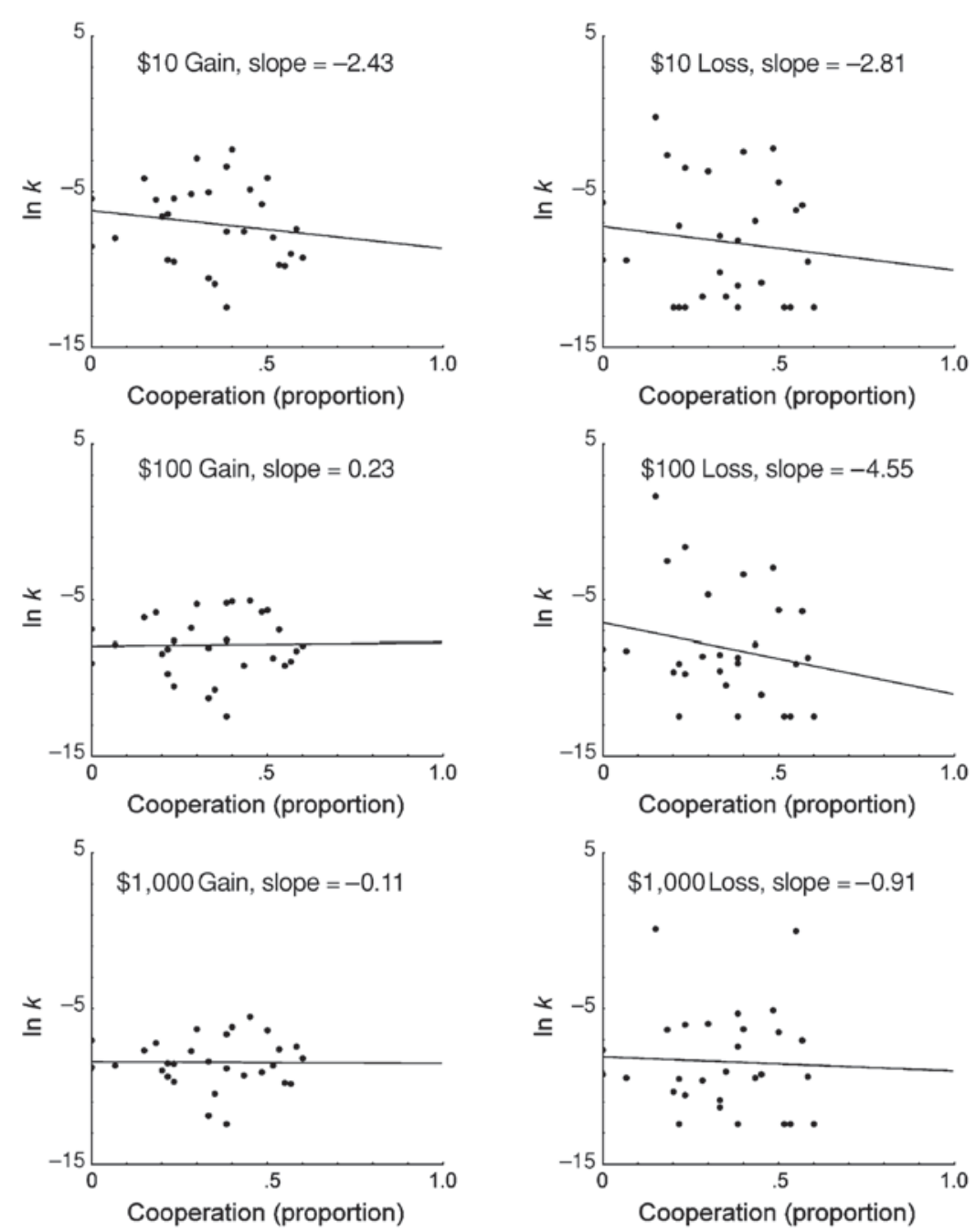

Figure 4. Scatterplots of natural logarithm transformed $k$ as a function of proportion of cooperation against the random strategy, with linear regression lines.

delay-discounting rate. Cooperation in IPG against a TFT strategy is similar to self-control because both require the participant to consistently delay immediate reinforcement for the largest overall outcome. Against RAN, outcomes are maximized by defection, rather than by cooperation. Therefore, a significant relationship between performance in an IPG against any strategy and the delay-discounting rate would suggest that high discounters have a generalized tendency to either cooperate or defect in an IPG, regardless of the opponent's strategy. Although the moderate correlations between performance measures in the two sessions indicate that some individuals have some generalized tendency to cooperate in an IPG, this tendency is not correlated with discount rate (as was indicated in the RAN condition). A significant relationship between the cooperation rate in the RAN condition and the delaydiscounting rate would have been a profoundly different (although interesting) finding from that proposed by Ainslie and by Rachlin.
Unfortunately, available IPG strategies that can serve as a control to TFT (e.g., RAN, always cooperate, always defect) will necessarily lead to lower cooperation rates (Silverstein, Cross, Brown, \& Rachlin, 1998). Given the generally low rates of cooperation observed in the IPG against RAN, the nonsignificant correlations with the discounting rate might be a result of the range of cooperation rates observed; most points in Figure 4 fall toward the middle and left in all the panels. Although visual inspection and a comparison of Figures 3 and 4, as well as the near-zero correlations in Table 2, suggest that such an alternative explanation is not likely, the presence of a unique relationship between performance in IPG against TFT and delay discounting cannot be stated with certainty.

The present results also extend the findings of Harris and Madden (2002) by changing the order in which the IPG and the delay-discounting procedures were conducted. The IPG always preceded the delay-discounting procedure in the present study. The opposite was true in 
Harris and Madden, and this difference is relevant to the concern that the previously obtained results could have been specific to that particular order of presentation. In addition, the present study showed that the relationship between dependent measures in the two procedures was maintained with different magnitudes and signs in the delay-discounting procedure. Harris and Madden obtained a significant correlation with only one magnitude (potentially real $\$ 10$ for the delayed reward) in the discounting procedure for gains. The present study, using hypothetical rewards, showed the same relationship across different magnitudes and signs - specifically, discounting parameters for larger magnitudes for both gains and losses. Interestingly, correlations between performance in the IPG against TFT and delay-discounting parameters were consistently higher for losses than for gains. This could be a new insight into the relationship between performance in IPG against TFT and delay-discounting parameters beyond that introduced by Harris and Madden. It remains to be seen whether this stronger correlation between the IPG against TFT and discounting for losses will be reflected in future studies.

Neither age nor intelligence was significantly correlated with performance in the IPG. Assuming that the goal of most players in the IPG was to earn the most money possible (particularly when the other player was a computer), there could have been an element of intellectual functioning that influenced a player's ability to maximize overall reinforcement. Intelligence, as measured by the QT in the present study, was not significantly correlated with performance in the IPG against the TFT, although the relationship was in the predicted direction. Although the highest scorers on the QT were likely to have extensive vocabularies, those who maximized total earnings in the IPG against the TFT (accomplished by cooperating) were likely both to have detected the pattern of choices made by the computer and to have determined the strategy that would maximize total earnings. Given these properties, the IPG may share more in common with tests of intelligence that measure some aspect of problem-solving ability (e.g., the performance scales of the WAIS), rather than strictly vocabulary or verbal intelligence. Although no evidence of a relationship between performance in the IPG and intelligence was observed in the present study, this may have been a function of the intelligence measure selected.

Finally, a discussion about the poor fit of the present data to Mazur's (1987) hyperbolic discounting equation is necessary. Harris and Madden (2002) reported a goodness-of-fit measure to the median indifference points $\left(r^{2}=.89\right)$ that was substantially higher than most of those shown in Figure 2. Two procedural dissimilarities between the studies provide possible explanations for the disparity. First is the use of real rewards in Harris and Madden. Although no evidence of differences has been found in comparisons of data obtained for real and hypothetical rewards in discounting (Johnson \& Bickel, 2002; Madden,
Begotka, Raiff, \& Kastern, 2003; Madden et al., 2004), it is possible that participants attend more to choices when real money is involved, thus providing less noisy data. Indeed, similar discounting procedures (Baker et al., 2003) that have obtained better fits included real gains, as well as compensation for participants. The second important difference is Harris and Madden's use of a card task procedure in which the immediate reward strictly increased (or decreased) within a block of trials. Two indifference points were obtained at each delay (from increasing and decreasing immediate reward conditions), and the obtained indifference point was calculated as the mean of the two. Not only is this transparent procedure likely to result in greater consistency from block to block (delay to delay), but also the use of two indifference points would reduce the effects of any single anomalous choice.

Although the measures of goodness of fit of the obtained data to Mazur's (1987) equation are lower than expected, we do not believe that this should invalidate the observations of the present study. Independently of $r^{2}$, low indifference points result in high parameters, and high indifference points result in low parameters with Mazur's equation. Furthermore, significant between-sessions correlations and the inclusion of AUC measures confirmed the reliability/validity of discounting parameters. Discounting parameters and AUC measures were all highly correlated (all greater than - .93), and analyses including performance measures in the IPG revealed nearly identical effects with both measures of discounting.

\section{EXPERIMENT 2}

Mazur's (1987) hyperbolic delay-discounting equation has been widely accepted in behavior analysis as a good descriptor of the rate of delay discounting, and a similar hyperbolic discounting equation (Rachlin, Raineri, \& Cross, 1991) has been shown to account for much of the variance with probability discounting:

$$
v_{\mathrm{d}}=\frac{V}{1+h \theta} \text {. }
$$

In this equation, the discounted value of an outcome $\left(v_{\mathrm{d}}\right)$ is equal to the ratio of the undiscounted value $(V)$ to the quantity one plus the odds against $[\theta$ : equal to $(1-$ probability) / probability] multiplied by a discounting parameter $(h)$. This free parameter $(h)$ provides a measure of the tendency to prefer smaller, certain rewards to larger, probabilistic outcomes. High $h$ values indicate a greater reduction in subjective value, resulting from uncertainty (more discounting). Low $h$ values indicate little reduction in subjective value (less discounting), with a value of zero indicating no discounting at all.

The results from numerous discounting studies suggest that delay discounting and probability discounting are related. Myerson, Green, Hanson, Holt, and Estle (2003) and Richards, Zhang, Mitchell, and de Wit (1999) have found positive correlations between the two forms of discounting. 
Furthermore, gamblers/nongamblers (Holt, Green, \& Myerson, 2003) and smokers/nonsmokers (Reynolds, Richards, Horn, \& Karraker, 2004) have been differentiated on parameters obtained from both delay- and probabilitydiscounting procedures. Given the significant correlations between delay-discounting parameters and performance in the IPG in Experiment 1, it stands to reason that similar results should be observed when probability discounting is correlated with IPG performance. The purpose of the present experiment was to examine this relationship in a manner identical to that in Experiment 1.

\section{Method}

Participants. Thirty college students, between 18 and 23 years of age, were initially recruited. Two participants failed to complete both sessions, and 2 participants were accidentally run in the wrong condition in one session; they were excluded from all analyses. The mean age of the remaining 26 participants ( 8 of them male) was 18.9 years. All the participants were recruited from the psychology department participant pool and received credit in an introductory-level class for participation.

Apparatus and Instruments. The QT (Ammons \& Ammons, 1962) was administered to assess intelligence. Both the IPG and the probability-discounting measures were obtained using a laptop computer running Visual Basic 6.0.

Procedure. The procedure was identical to that in Experiment 1, with the exception of probabilities replacing delays in the discounting procedure. Indifference points were obtained for $\$ 10, \$ 100$, and $\$ 1,000$ hypothetical standard gains, at each of the following probabilities: $99 \%, 90 \%, 75 \%, 60 \%, 40 \%, 25 \%, 10 \%$, and $1 \%$. These probabilities were always presented in decreasing order. The same order and procedure were then applied with the hypothetical outcomes for losses. An attempt was made to counterbalance the order in which the three magnitudes were presented (ascending and descending) with order of IPG strategy (TFT or RAN first), although perfect counter- balancing was not accomplished because of sample size and the loss of participants. The probability-discounting procedures in the two experimental sessions were identical in all respects.

\section{Results}

Mean proportion of cooperation was higher against TFT (.49) than against RAN $[.30 ; F(1,25)=16.61, p<.05]$. These proportions were significantly different in the first half of the session $[M=.45$ and .34 for TFT and RAN, respectively; $F(1,25)=4.77, p<.05]$ and in the second half of the session $[M=.53$ and .26 for TFT and RAN, respectively; $F(1,25)=18.70, p<.05]$. The hypothetical earnings against TFT $(M=\$ 896.11)$ and RAN $(M=$ $\$ 976.11)$ were significantly different $[F(1,25)=4.86$, $p<.05]$. Table 3 shows Pearson correlations between age, QT score, and proportion of cooperation and money earned against each strategy. Gender was not significantly correlated with any dependent variables. QT score was not significantly correlated with any measure obtained from the IPG. Age, however, was significantly and positively correlated with proportion of cooperation when the opponent used the TFT strategy. As in Experiment 1, the correlation between proportion of cooperation in the TFT and the RAN conditions was significant and positive.

Nonlinear estimation using Statistica 5.0 was used to determine probability-discounting parameters $(h)$ with median indifference points according to Equation 2 (Figure 5). Natural logarithm transformations were conducted on individual discounting parameters to normalize the distributions, and order effects were ruled out. Although $r^{2} \mathrm{~s}$ were very high, AUC measures were also calculated to maintain continuity with Experiment 1. Spearman cor-
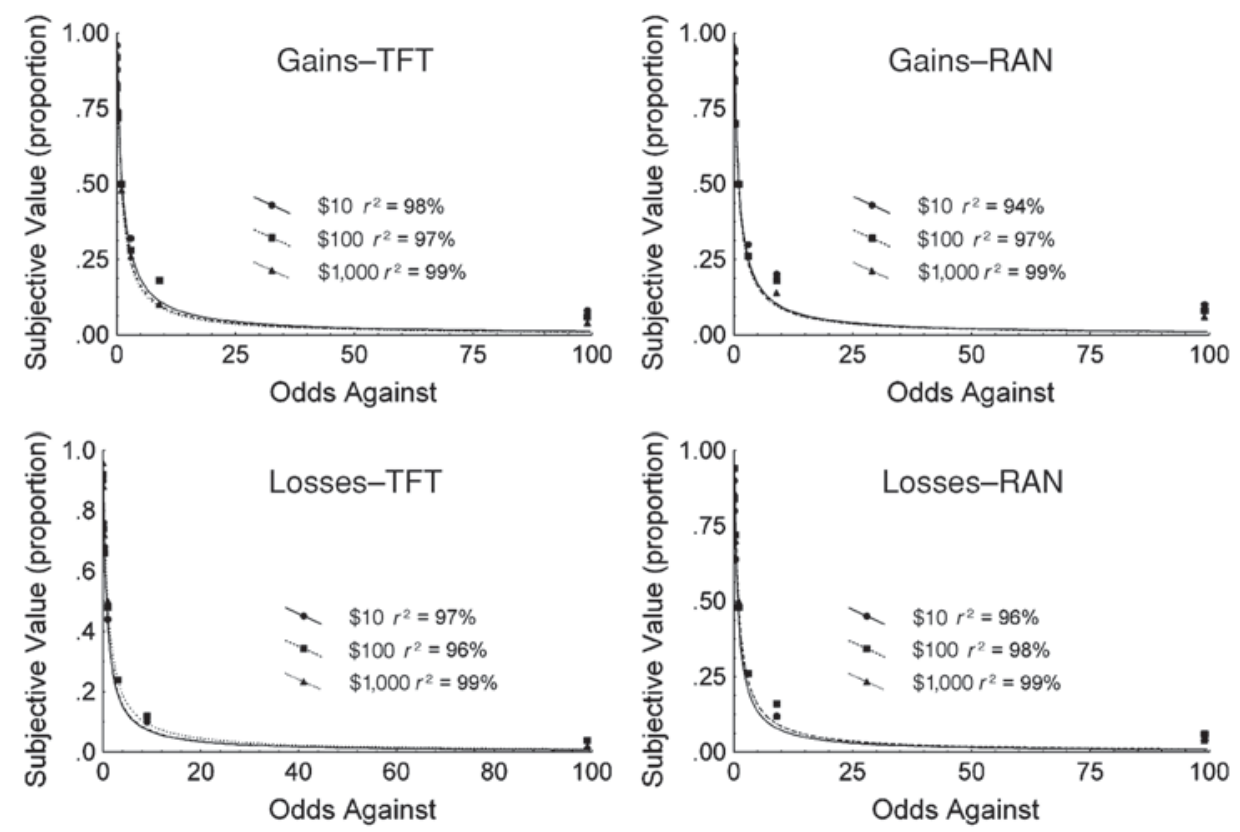

Figure 5. Median indifference points fitted to Equation 2. The graphs show (clockwise from top left) gains in the tit-for-tat (TFT) strategy, gains in the random (RAN) strategy, losses in the RAN strategy, and losses in the TFT strategy. Different magnitudes are represented in each graph. 

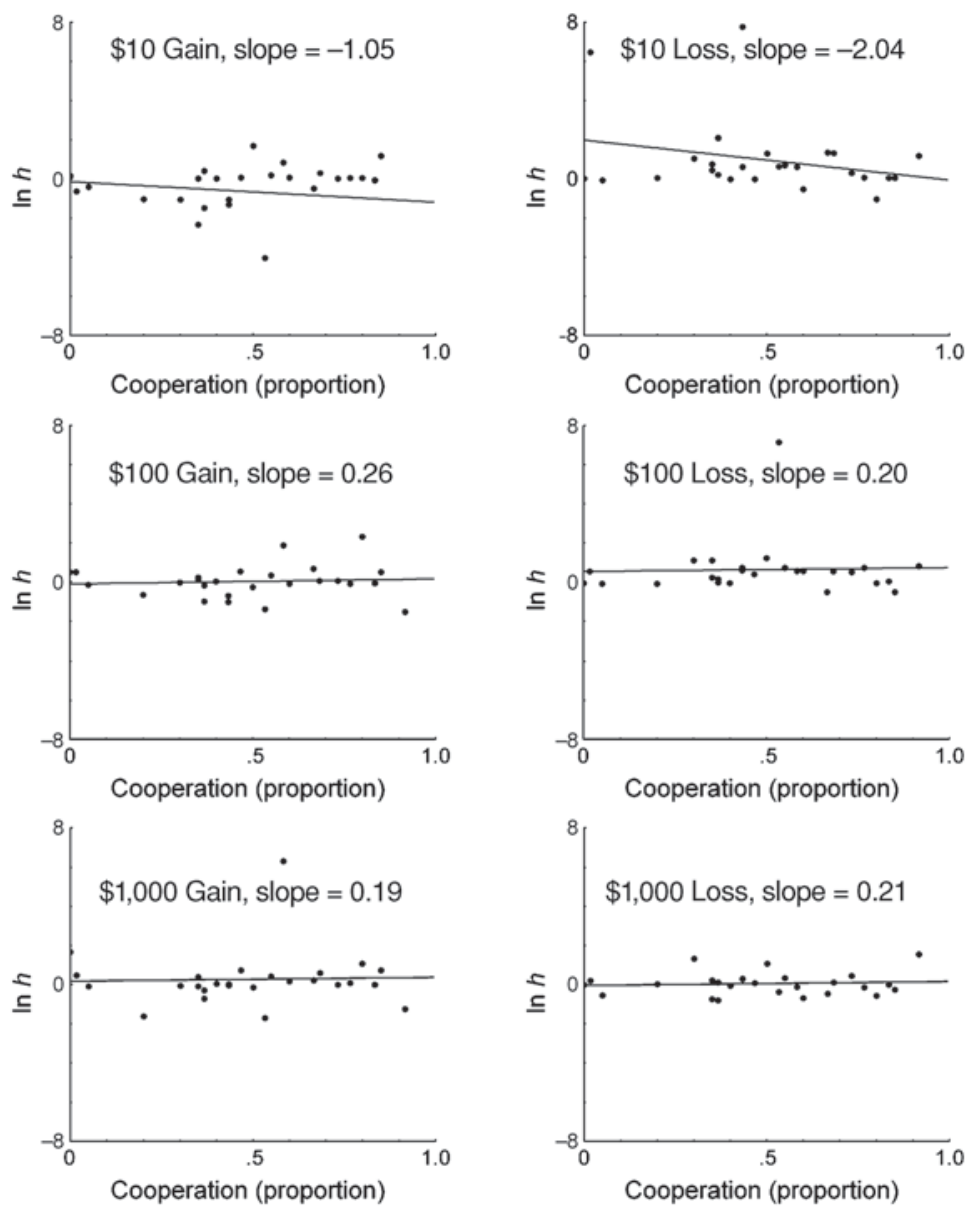

Figure 6. Scatterplots of natural logarithm transformed $\boldsymbol{h}$ as a function of proportion of cooperation against the tit-for-tat strategy, with linear regression lines.

relations between discounting parameters and AUC were moderate to high, with a range of -.26 to -.78 . All the correlations were significant at $p<.05$, with the exception of the $\$ 10$ loss in TFT and the $\$ 100$ loss in RAN.

Like Experiment 1, order effects of IPG condition were ruled out, and the remaining analyses pooled data across order of IPG. A repeated measures ANOVA revealed no difference between probability-discounting parameters

Table 3

Pearson Correlations Between Demographic Variables and Iterated Prisoner's Dilemma Game Data (Experiment 2)

\begin{tabular}{|c|c|c|c|c|c|c|}
\hline & \multirow[b]{2}{*}{ Age } & \multirow{2}{*}{$\begin{array}{c}\text { Quick } \\
\text { Test }\end{array}$} & \multicolumn{2}{|c|}{ TFT } & \multicolumn{2}{|c|}{ RAN } \\
\hline & & & Earn & Coop & Earn & Coop \\
\hline Age & - & .19 & .42 & $.40^{*}$ & .01 & -.08 \\
\hline Quick test & - & - & .30 & .29 & -.33 & .15 \\
\hline \multicolumn{7}{|l|}{ TFT } \\
\hline Earn & - & - & - & $.94^{*}$ & -.36 & .36 \\
\hline Coop & - & - & - & - & -.22 & $.42^{*}$ \\
\hline \multicolumn{7}{|l|}{ RAN } \\
\hline Earn & - & - & - & - & - & $-.64^{*}$ \\
\hline Coop & - & - & - & - & - & - \\
\hline
\end{tabular}

Note-TFT, tit-for-tat strategy; RAN, random strategy; Earn, money earned; Coop, proportion of cooperation. ${ }^{*} p<.05$. resulting from IPG condition $[F(1,25)=0.73, p>.05]$, sign $[F(1,25)=1.55, p>.05]$, or magnitude $[F(2,50)=$ $0.04, p>.05]$. However, large-magnitude outcomes were discounted more than small-magnitude outcomes when only gains were included in a planned comparison $[F(2,50)=$ $5.19, p>.05]$, with a significant difference $(p<.05)$ between $\$ 10(M=-0.42)$ and $\$ 1,000(M=0.26)$ outcomes. The same analysis with AUC revealed somewhat different results. Although differences due to the IPG $[F(1,25)=$ $0.51, p>.05]$ and sign conditions $[F(1,25)=2.93, p>$ $.05]$ were nonsignificant, the reverse magnitude effect was observed $[F(2,50)=14.92, p<.05]$, with significant differences between $\$ 10(M=18.05)$ and the remaining magnitudes $(\$ 100, M=14.82 ; \$ 1,000, M=12.72)$. The nonsignificant effect of sign was in the predicted direction, with $p=.06$.

Spearman correlations were conducted between discounting parameters in the TFT and RAN conditions. Although all the correlations were in the predicted direction (positive), only that from the $\$ 100$ loss condition was statistically significant. The same analysis with AUC revealed more mixed results: Correlations from the gains condition at all magnitudes were between .49 and .60 and 

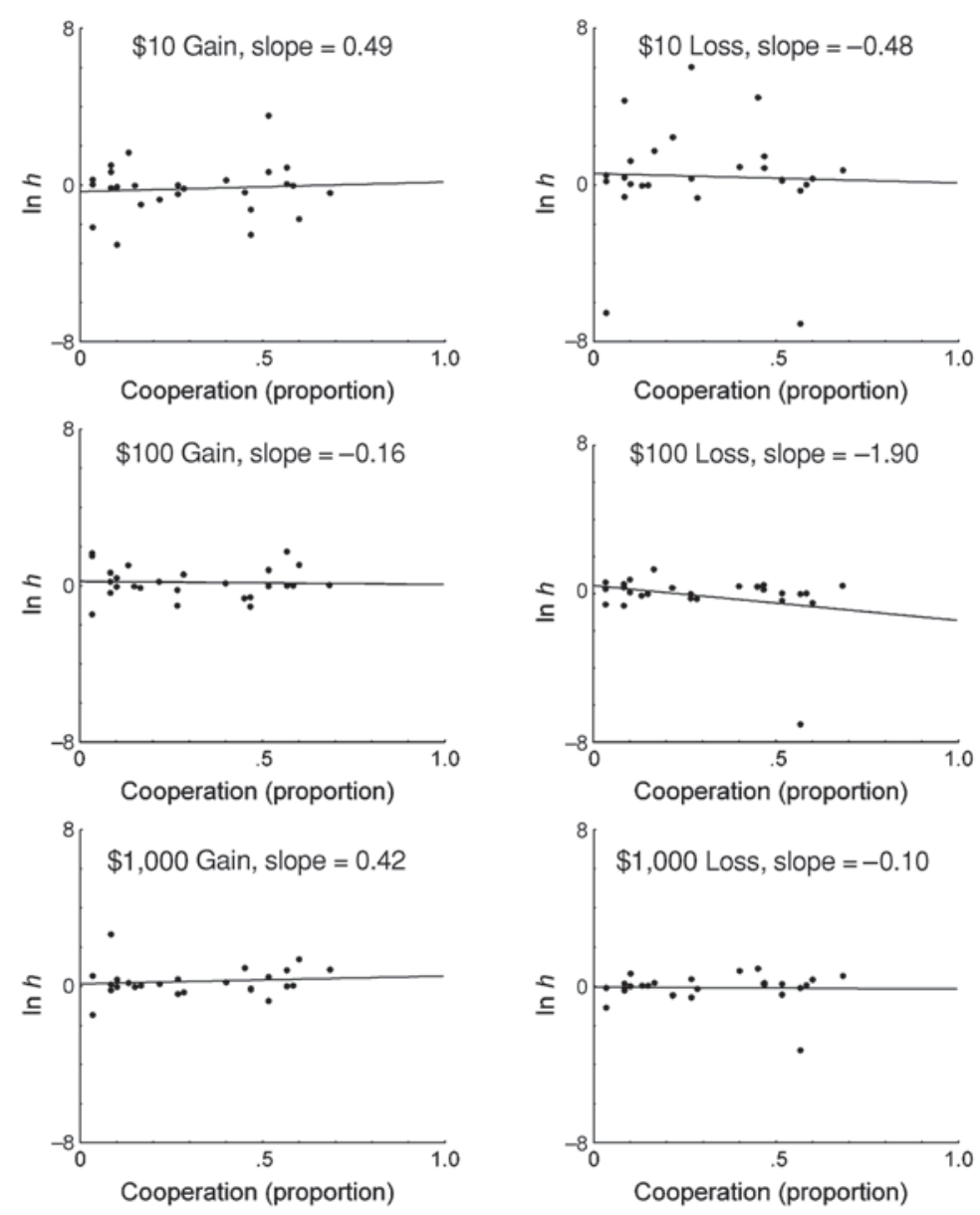

Figure 7. Scatterplots of natural logarithm transformed $h$ as a function of proportion of cooperation against the random strategy, with linear regression lines.

were significant at $p<.05$. Correlations for losses were all fairly low and nonsignificant. These analyses, taken together, indicate little consistency in probability discounting.

Spearman correlations were calculated between performance measures in the IPG and probability-discounting parameters. Table 4 displays the correlation matrix between these measures obtained in the TFT and RAN conditions. Figure 6 displays scatterplots of proportion of cooperation and the natural logarithm of the discounting parameter $(\ln$ $h$ ) for gains and losses in the TFT condition. Linear regression lines were fit to the data, and slopes are reported. Figure 7 displays scatterplots of proportion of cooperation and $\ln h$ for gains and losses in the RAN condition. Correlations with probability-discounting parameters and performance in the IPG were significant in neither the TFT nor the RAN condition. The same pattern of results was obtained with AUC data, with no significant correlations between IPG performance and AUC measures.

\section{Discussion}

Some results from the present experiment are similar to those from Experiment 1. They include a significant cor- relation between rates of cooperation against the TFT and RAN strategies in the IPG. Also, no difference in discounting could be attributed to IPG condition. The present results are different in many other ways from those obtained in Experiment 1 . Correlations between discounting parameters between the IPG conditions were largely nonsignificant. This indicates that probability discounting is not as stable a phenomenon as delay discounting. We are unaware of any studies that have examined the consistency of probabilitydiscounting measures, and therefore, it is unclear whether the nonsignificant correlations between parameters are unique to this data set or a real phenomenon.

The absence of the reverse magnitude effect in the overall analysis may also pose a challenge to the accuracy of this data set. However, this may be a function of a procedural and a statistical difference. In studies that have shown the reverse magnitude effect, a range of magnitudes far greater than that in the present study has been used. For instance, Green, Myerson, and Ostaszewski (1999) obtained the reverse magnitude effect with $\$ 200, \$ 5,000$, and \$100,000 magnitudes. Given the smaller range of magnitudes in the present study, the results may not be inconsistent. The sta- 
Table 4

Spearman Correlations Between Iterated Prisoner's Dilemma Game Performance and Probability-Discounting Parameters

\begin{tabular}{lrcc}
\hline Condition & $\begin{array}{c}\text { Total } \\
\text { Coop }\end{array}$ & $\begin{array}{c}\text { Trials 1-30 } \\
\text { Coop }\end{array}$ & $\begin{array}{c}\text { Trials 31-60 } \\
\text { Coop }\end{array}$ \\
\hline TFT & & & \\
\$10 gains & .23 & .17 & .16 \\
\$100 gains & .06 & .03 & .06 \\
\$1,000 gains & .17 & .14 & .09 \\
\$10 losses & -.08 & -.07 & -.05 \\
\$100 losses & .07 & -.01 & .15 \\
\$1,000 losses & -.02 & .01 & -.02 \\
RAN & & & \\
\$10 gains & -.05 & .03 & -.07 \\
\$100 gains & -.02 & .03 & -.09 \\
\$1,000 gains & .12 & .11 & .09 \\
\$10 losses & -.00 & .00 & .01 \\
\$100 losses & -.20 & -.11 & -.24 \\
\$1,000 losses & .25 & .27 & .20 \\
\hline
\end{tabular}

Note-TFT, tit-for-tat; RAN, random; Coop, proportion of cooperation.

tistical difference (which is also a procedural difference) is the inclusion of a discounting-for-loss condition. To the best of our knowledge, previous studies on probability discounting have included only positive outcomes. Given the presence of the reverse magnitude effect in the present study when losses were excluded, the results are consistent with those in the previous literature.

Unlike the results from Experiment 1, performance in the IPG against the TFT strategy and rate of probability discounting were not correlated. This is somewhat surprising, given previously observed significant correlations between delay-discounting and probability-discounting measures (Myerson et al., 2003; Richards et al., 1999). Although cooperation rate in IPG against the TFT strategy and delay-discounting parameters (in Experiment 1) were all negatively correlated (and mostly significant), this was not the case with probability-discounting parameters. Because the $1 \%$ probability (99 odds against in Figure 5) had a relatively large influence in the calculation of the discounting parameter, the variability of $h$ values may have been reduced and may have attenuated the relationship with performance in the IPG (Figure 6). To address this possibility, new discounting parameters were calculated for each participant in each condition without indifference points from the $1 \%$ condition. No differences in discounting rate were observed, and more important, variance measures were nearly identical. Similar results were observed with AUC measures. The lack of relationship between probability discounting and IPG performance does not appear to be a result of restricted range. This indicates that IPG play against the TFT strategy and probability-discounting procedures do not share a fundamental behavioral process.

As was proposed by Ainslie (1992) and Rachlin (2000), delay-discounting procedures and IPG against the TFT strategy both require participants to reject the larger of two available alternatives to maximize overall outcome. Against the strict version of TFT employed in the present study, where probability of reciprocation is equal to 1 , reinforcement for cooperation is always delayed by one trial; there is no probabilistic component about the choice of the computer on trial $n+1$. Like delay-discounting procedures, reinforcement always follows a delay. On the other hand, there is no delay in probability-discounting procedures, and with the probabilistic component, choice for the larger, probabilistic option would at times result in no reinforcement at all. This is unlike the IPG of the present study and more similar to the IPG employed by Baker and Rachlin (2001), where the probability of reciprocation ranged from .5 to 1 . This further suggests that individuals do not convert probabilities to waiting times, contrary to the proposition of Rachlin et al. (1991). If individuals did convert probabilities to waiting times, probability discounting would be functionally similar to delay discounting, and similar results would have been found.

Similar to the results from Experiment 1, no significant relationships were observed between performance in the IPG and demographic variables such as age (with one exception) and intelligence (as measured by the QT). Although delay-discounting procedures have differentiated age groups, we are unaware of studies in which performance in the IPG has been examined as a function of age. Furthermore, the age ranges in studies that have shown age differences in discounting (e.g., Green, Myerson, Lichtman, Rosen, \& Fry, 1996) were far more extensive than that in the present study. Given that all the participants in the present study were college students with a 5year age range, it is not surprising to find nonsignificant correlations between age and measures in the IPG. As in Experiment 1, correlations between intelligence and performance measures were also nonsignificant. Once again, this could be a function of the intelligence measure selected, and a significant relationship between performance in the IPG and intelligence may be more likely with a more appropriate measure of intelligence.

\section{CONCLUSIONS}

Discounting rates, resulting from delay and probability, were correlated with performance in an IPG. Participants who were likely to cooperate when the opponent used a tit-for-tat strategy exhibited more self-control in a delaydiscounting procedure. The same relationship was not observed with a probability-discounting procedure. Importantly, behavior against a nonreciprocal (RAN) IPG strategy was correlated with neither delay- nor probabilitydiscounting procedures. This suggests that participants are sensitive to the reward contingencies (on the basis of opponent's strategy), and low discounters are able to delay gratification in an IPG against the TFT strategy. The significant correlation between rates of cooperation in the two IPG conditions does indicate the possibility of some generalized tendency to cooperate independently of IPG strategy, although the possibility that learning from the first IPG session influenced the rate of cooperation in the second session is also a reasonable explanation. 
A primary weakness of the present experiments was the use of hypothetical rewards. Harris and Madden (2002) used real rewards in the IPG (or at least the possibility of them), as well as the delay-discounting procedure. As was stated earlier, this could have resulted in the poor goodnessof-fit measures in the present study. Given the range of magnitudes, as well as the inclusion of a loss condition, real rewards were neither feasible nor possible in the present experiments. Nonetheless, the nature of the rewards may make an important difference in the IPG. An additional weakness of the present analyses was the use of different participants across the two experiments. A completely repeated measures design with both delay- and probabilitydiscounting procedures would have allowed for a more thorough analysis of the relationship between discounting and IPG, as well as the relationship between delay and probability. However, given that a major rationale for this study was the inclusion of the RAN condition, a similar repeated measures study would have required four experimental sessions with IPG and discounting procedures in each session. This was avoided because data from pilot studies involving delay-discounting procedures indicated that multiple exposures to discounting procedures lead to reductions in discounting parameters to near-floor levels, with no apparent explanation other than participant fatigue. Different participants for the two experiments were required in order to avoid this.

Regardless of these weaknesses, the present experiments confirm and extend the findings of Harris and Madden (2002), who found a significant correlation between cooperation rate in an IPG against the TFT strategy and delay-discounting rate; the same relationship was found across a range of magnitudes and for both gains and losses. Most important, the inclusion of a RAN condition, in which delayed reinforcement did not follow cooperation, led to the rejection of the possibility that a low rate of delay discounting could be correlated with a generalized tendency to cooperate in an IPG. Although some authors have suggested a functional equivalence between delay and probability discounting (Navarick, 1987; Rachlin et al., 1991; Stevenson, 1986) and significant correlations have been found between the measures, no significant correlations were found between probability-discounting rates and performance in an IPG.

\section{REFERENCES}

Ainslie, G. (1992). Picoeconomics. New York: Cambridge University Press.

Ammons, R. B., \& Ammons, C. H. (1962). Quick Test. Missoula, MT: Psychological Test Specialists.

BAKer, F., Johnson, M. W., \& Bickel, W. K. (2003). Delay discounting in current and never-before cigarette smokers: Similarities and differences across commodity, sign, and magnitude. Journal of Abnormal Psychology, 112, 382-392.

BAKER, F., \& Rachlin, H. (2001). Probability of reciprocation in repeated prisoner's dilemma games. Journal of Behavioral Decision Making, 14, 51-67.
Bickel, W. K., Odum, A. L., \& Madden, G. J. (1999). Impulsivity and cigarette smoking: Delay discounting in current, never, and ex-smokers. Psychopharmacology, 14, 447-454.

Coffey, S. F., Gudleski, G. D., Saladin, M. E., \& Brady, K. T. (2003). Impulsivity and rapid discounting of delayed hypothetical rewards in cocaine-dependent individuals. Experimental \& Clinical Psychopharmacology, 11, 18-25.

Crean, J. P., de Wit, H., \& Richards, J. B. (2000). Reward discounting as a measure of impulsive behavior in a psychiatric outpatient population. Experimental \& Clinical Psychopharmacology, 8, 155-162.

Green, L., Fry, A. F., \& Myerson, J. (1994). Discounting of delayed rewards: A life-span comparison. Psychological Science, 5, 33-36.

Green, L., \& Myerson, J. (2004). A discounting framework for choice with delayed and probabilistic rewards. Psychological Bulletin, 130, 769-792.

Green, L., Myerson, J., Lichtman, D., Rosen, S., \& Fry, A. (1996). Temporal discounting in choice between delayed rewards: The role of age and income. Psychology \& Aging, 11, 79-84.

Green, L., Myerson, J., \& Ostaszewski, P. (1999). Amount of reward has opposite effects on the discounting of delayed and probabilistic outcomes. Journal of Experimental Psychology: Learning, Memory, \& Cognition, 25, 418-427.

HaRris, A. C., \& MADDEN, G. J. (2002). Delay discounting and performance on the prisoner's dilemma game. Psychological Record, 52, 429-440.

Holt, D. D., Green, L., \& Myerson, J. (2003). Is discounting impulsive? Evidence from temporal and probability discounting in gambling and non-gambling college students. Behavioural Processes, 64, 355-367.

Joesting, J., \& Joesting, R. (1971). Comparison of scores on Quick Test and Stanford-Binet, Form L-M. Psychological Reports, 29, 1178.

JoHnson, M. W., \& BicKeL, W. K. (2002). Within-subject comparison of real and hypothetical rewards in delay discounting. Journal of the Experimental Analysis of Behavior, 77, 129-146.

Kirby, K. N., Petry, N. M., \& BicKel, W. K. (1999). Heroin addicts have higher discount rates for delayed rewards than non-drug-using controls. Journal of Experimental Psychology: General, 128, 78-87.

Kollins, S. H. (2003). Delay discounting is associated with substance use in college students. Addictive Behaviors, 28, 1167-1173.

Madden, G. J., BegotKa, A. M., RaifF, B. R., \& Kastern, L. L. (2003). Delay discounting of real and hypothetical rewards. Experimental \& Clinical Psychopharmacology, 11, 139-145.

Madden, G. J., Petry, N. M., Badger, G. J., \& Bickel, W. K. (1997). Impulsive and self-control choices in opioid-dependent patients and non-drug-using control participants: Drug and monetary rewards. Experimental \& Clinical Psychopharmacology, 5, 256-262.

Madden, G. J., Raiff, B. R., Lagorio, C. H., Begotka, A. M., MuelLer, A. M., Hehli, D. J., \& Wegener, A. A. (2004). Delay discounting of potentially real and hypothetical rewards: II. Between- and within-subject comparisons. Experimental \& Clinical Psychopharmacology. 12, 251-261.

Mazur, J. (1987). An adjusting procedure for studying delayed reinforcement. In M. Commons, J. Mazur, J. Nevin, \& H. Rachlin (Eds.), The effect of delay and of intervening events on reinforcement value (pp. 55-73). Hillsdale, NJ: Erlbaum.

Mitchell, S. H. (1999). Measures of impulsivity in cigarette smokers and non-smokers. Psychopharmacology, 146, 455-464.

Myerson, J., Green, L., Hanson, J. S., Holt, D. D., \& Estle, S. J. (2003). Discounting delayed and probabilistic rewards: Processes and traits. Journal of Economic Psychology, 24, 619-635.

Myerson, J., Green, L., \& Warusawitharana, M. (2001). Area under the curve as a measure of discounting. Journal of the Experimental Analysis of Behavior, 76, 235-243.

NAVARICK, D. J. (1987). Reinforcement probability and delay as determinants of human impulsiveness. Psychological Record, 37, 219-226.

Petry, N. M. (2001a). Delay discounting of money and alcohol in actively using alcoholics, currently abstinent alcoholics, and controls. Psychopharmacology, 154, 243-250. 
Petry, N. M. (2001b). Pathological gamblers, with and without substance abuse disorders, discount delayed rewards at high rates. Journal of Abnormal Psychology, 110, 482-487.

Rachlin, H. (2000). The science of self-control. Cambridge, MA: Harvard University Press.

Rachlin, H., Raineri, A., \& Cross, D. (1991). Subjective delay and probability. Journal of the Experimental Analysis of Behavior, 55, 233-244.

Reynolds, B., Richards, J. B., Horn, K., \& Karraker, K. (2004). Delay discounting and probability discounting as related to cigarette smoking status in adults. Behavioural Processes, 65, 35-42.

Richards, J. B., Zhang, L., Mitchell, S. H., \& DE Wit, H. (1999). Delay or probability discounting in a model of impulsive behavior: Effect of alcohol. Journal of the Experimental Analysis of Behavior, 71, 121-143.
Silverstein, A., Cross, D., Brown, J., \& Rachlin, H. (1998). Prior experience and patterning in a prisoner's dilemma game. Journal of Behavioral Decision Making, 11, 123-138.

Stevenson, M. K. (1986). A discounting model for decisions with delayed positive or negative outcomes. Journal of Experimental Psychology: General, 115, 131-154.

Vuchinich, R. E., \& Simpson, C. A. (1998). Hyperbolic temporal discounting in social drinkers and problem drinkers. Experimental \& Clinical Psychopharmacology, 6, 292-305.

(Manuscript received November 17, 2004; revision accepted for publication January 7, 2005.) 